$\mathrm{P}$

ОАь поражения энАОтеАия и Аислипидемий в развитии кардиоваскулярной патологии при псориатическом артрите

(C) Хисматуллина 3.P., Корешкова К.М.*

Башкирский государственный медицинский университет

450008, Россия, Республика Башкортостан, г. Уфа, ул. Ленина, д. 3

В настоящее время установлен повышенный риск сердечно-сосудистых осложнений у пациентов, страдающих псориатическим артритом (ПА). Хроническое иммунное воспаление, лежащее в основе ПА, приводит к ускорению развития дислипидемии, атеросклероза и его осложнений, в частности, к высокому риску кардиоваскулярных событий. Для ПА наиболее характерны дислипидемии, проявляющиеся повышенными уровнями липопротеидов низкой и очень низкой плотности, триглицеридов и общего холестерина, коррелирующими с активностью заболевания. В литературном обзоре изучены патогенез дислипидемий и поражения сосудистой стенки при псориатическом артрите, проведен анализ литературных источников о кардиоваскулярных осложнениях и смертности среди пациентов с ПА, изучены данные по суммарному сердечно-сосудистому риску, приведены результаты многочисленных клинических исследований, позволяющие считать ПА заболеванием, с которым ассоциируется повышенный риск сердечно-сосудистых осложнений. Раннее выявление эндотелиального поражения представляет собой наиболее перспективное направление в предупреждении кардиоваскулярных заболеваний, которые являются основной причиной летальности у данной группы больных.

Ключевые слова: псориатический артрит, дислипидемии, атеросклероз, кардиоваскулярные осложнения.

Конфрликт интересов: авторы данной статьи подтвердили отсутствие конфликта интересов, о котором необходимо сообщить.

Источник финансирования: работа выполнена и опубликована за счет финансирования по месту работы авторов.

Для цитирования: Хисматуллина З.Р., Корешкова К.М. Роль поражения эндотелия и дислипидемий в развитии кардиоваскулярной патологии при псориатическом артрите. Вестник дерматологии и венерологии. 2021;97(2):16-22. doi: https://doi.org/10.25208/vdv1206 


\title{
1 he role of endothelial damage and dyslipidemia in the development of cardiovascular pathology in psoriatic arthritis
}

\author{
(C) Zarema R. Khismatullina, Ksenia M. Koreshkova*
}

Bashkir State Medical University

Lenina str., 3, 450008, Ufa, Republic of Bashkortostan, Russia

An increased risk of cardiovascular events has now been identified in patients with psoriatic arthritis. The chronic immune-mediated inflammation underlying psoriatic arthritis (PA) leads to the development of dyslipidemia, atherosclerosis and its complications, in particular, a high risk of cardiovascular complications. For PA, dyslipidemias are most characteristic, manifested by an increased level of low and very low density lipoproteins, triglycerides and total cholesterol, which correlates with the activity of the disease. The literature review studied the pathogenesis of dyslipidemias and vascular wall lesions in psoriatic arthritis, analyzed the literature on cardiovascular complications and mortality among patients with $\mathrm{PA}$, studied the issues of total cardiovascular risk, presented the results of numerous clinical studies that allow PA to be considered a disease associated with increased the risk of cardiovascular complications. Considering the role of proinflammatory cytokines in the pathogenesis of psoriatic arthritis, early detection of endothelial lesions represents the most promising direction in the prevention of cardiovascular diseases, which are the main cause of mortality in this group of patients.

Keywords: psoriatic arthritis, dyslipidemias, atherosclerosis, cardiovascular complications.

Conflict of interest: the authors of this article have confirmed that they have no conflict of interest to report.

Source of funding: the work was done and published through financing at the place of work of the authors.

For citation: Khismatullina ZR, Koreshkova KM. The role of endothelial damage and dyslipidemia in the development of cardiovascular pathology in psoriatic arthritis. Vestnik Dermatologii i Venerologii. 2021;97(2):16-22.

doi: https://doi.org/10.25208/vdv1206 
Псориатический артрит (ПА) представляет собой хроническое воспалительное заболевание из группы серонегативных периферических спондилоартритов. Частота встречаемости ПА среди лиц, страдающих псориазом, колеблется от 13,5 до 47,5\% [1]. В настоящее время ПА, в основе которого лежит хроническое иммунное воспаление, характеризуется как заболевание с высоким риском сердечно-сосудистых осложнений [1]. Данный обзор литературы посвящен определению основных звеньев патогенеза повреждения эндотелия, дислипидемии и их роли в развитии кардиоваскулярных осложнений у пациентов с ПА.

Был проведен анализ российской и зарубежной литературы, посвященной проблемам хронического иммунного воспаления при ПА, повреждениям сосудистой стенки (в особенности - интимы артерий) и сердечно-сосудистым осложнениям при данном заболевании. Были проанализированы научные публикации, вышедшие в период 2010-2020 гг., содержащие материалы по вышеуказанным темам. Литературные источники, вышедшие ранее 2010 г., в исследование не включались.

В российской и зарубежной литературе представлены результаты клинических исследований, выявляющие более высокую частоту заболеваемости и смертности от кардиоваскулярных событий у больных, страдающих ПА, в сравнении с лицами без данного заболевания [2]. Повреждение и следующая за этим дисфункция эндотелия как ответ на хроническое системное иммунное воспаление являются проявлением атеросклероза, осложнения которого приводят к преждевременной летальности у больных ПА [2]. По данным некоторых авторов, ПА сокращает ожидаемую продолжительность жизни в среднем на 3-4 года, при этом наиболее распространенными причинами летальности являются инфраркт миокарда и острое нарушение мозгового кровообращения (ОНМК). Среди женщин, страдающих данным заболеванием, смертность превышает популяционную на 59\%, среди мужчин на 65\% [3]. Считается, что кардиоваскулярные осложнения находятся в прямой зависимости от степени тяжести ПА и могут быть объяснены иммунным характером системного воспаления [3]. Согласно данным Рекомендаций Европейского общества кардиологов, пациенты с тяжелым течением ПА имеют более высокий риск сердечно-сосудистых событий [4].

\section{Актуальность проблемы}

По данным Е.И. Маркеловой и соавт. (2016г.), В структуре причин смертности при ПА кардиоваскулярные осложнения находятся на первом месте $(23,6 \%)$ и опережают онкозаболевания, болезни органов дыхания и желудочно-кишечного тракта [5]. Эти данные подтверждаются результатами крупного исследования E. Lihi и соавт. (2014 г.), согласно которым среди причин смертности при ПА на первое место выступили болезни системы кровообращения (32\%). Авторы исследования делают вывод о повышенной смертности среди больных с ПА в популяции, прогностическими факторами для которой выступают высокая активность заболевания, выражающаяся величиной СОЭ, СРБ и скоростью рентгенологической деструкции [6]. При нормальных показателях СОЭ смертность у больных составила $3,2 \%$, в то время как при СОЭ более 15 мм/ч - 17\%. Была выявлена ассоциация между скоростью костной деструкции и повышенной смертностью. Еще в одном исследовании (О.В. Дегтярев и соавт., 2015 г.) была выявлена наиболее высокая смертность больных ПА от сердечно-сосудистых событий, где 33\% летальных случаев приходилось на инфраркт миокарда и ОНМК [7]. D. D. Gladman (2019 г.) и соавт. в своем наблюдении о структуре кардиоваскулярной патологии отмечают инфраркт миокарда (28\%), ОНМК (4\%) и сердечную недостаточность (4\%), а также более высокую смертность от заболеваний системы кровообращения у больных ПА по сравнению с популяционным уровнем [8]. Таким образом, псориатический артрит ассоциируется с повышенным риском кардиоваскулярной смертности.

\section{Факторы риска сердечно-сосудистых осложнений при ПА}

Среди фракторов риска сердечно-сосудистых осложнений при ПА особенно выделяют отягощенную наследственность по сердечно-сосудистым заболеваниям (ССЗ), наличие артериальной гипертензии, дислипидемии и метаболического синдрома [9]. По данным M.H. Favarato и соавт. (2014), артериальная гипертензия встречается у 87,55\% больных ПА [9]. По данным И. 3. Гайдуковой и соавт. (2015 г.), данная патология обнаруживается в 53\% случаев, гиперхолестеринемия в 55\%, повышение уровня триглицеридов - в 14,4\% наблюдений соответственно [10]. C. Francesco и соавт. (2020 г.) считают ПА независимым фактором риска для артериальной гипертензии и сердечно-сосудистой смертности [11]. Важно отметить, что причиной липидных изменений могут быть препараты для лечения ПА, например, циклоспорин и ингибиторы ФНО-а, которые предположительно приводят к повышению уровня триглицеридов [12]. Особую роль в патогенезе сердечно-сосудистых осложнений играет то, что большая часть больных не получают рационального гипотензивного лечения [12].

\section{Липидные нарушения}

Изучению обмена холестерина при ПА уделяется особое внимание. Патология липидного спектра на сегодняшний день позволяет нам рассматривать псориаз в тесной связи с атеросклерозом [13]. По данным М.В. Ахлупкиной и соавт. (2011 г.), синтез холестерина и ЛПНП с последующим выбросом их в системный кровоток вызывается гиперпродукцией цитокинов и нарушением регуляции клеточного иммунитета, сопровождающих ПА [13]. Дислипидемия при ПА характеризуется повышением проатерогенных липидных фрракций. Изучение липидного спектра у больных ПА позволило обнаружить повышение уровня липопротеидов (ЛП) преимущественно низкой и очень низкой плотности, коррелирующее с длительностью заболевания, - уже на ранней стадии заболевания уровень ЛП был выше, чем в популяции, и нарастал с увеличением длительности ПА [14]. Повышение в сыворотке крови уровня общего холестерина выше верхней границы нормы, по данным некоторых авторов, при ПА широко варьирует - от 20 до 80\%, а наиболее часто встречающимися типами гиперлипидемий являются гиперлипопротеидемии IIA, ІІБ и IV типов, а также повышение уровня апопротеинов (что, предположительно, косвенно свидетельствует о тяжелом течении заболевания) [15]. По данным В. Kirby и соавт. (2020 г.), гиперхолестеринемия у больных ПА встречается 
в 2,5 раза чаще, чем в общей популяции (18 и 8\% соответственно), и нередко предшествует клинической манифестации артрита [16]. Некоторые данные также свидетельствуют об обнаружении значимых нарушений липидного профиля (увеличения уровня свободных жирных кислот, триглицеридов, общего холестерина) уже на ранней (субклинической) стадии ПА [17]. Так, C. Acebes и соавт. (2015 г.) провели исследование 200 больных ПА, не имеющих жалоб и симптомов сердечно-сосудистых заболеваний, и выявили повышение концентрации липопротеинов низкой плотности, не коррелирующее с уровнем С-реактивного белка, полом, давностью артрита или индексом распространенности и тяжести псориаза (PASI) [17]. В другом исследовании (W. Badaiki и соавт., 2019 г.) повышенный уровень аполипопротеина-В и триглицеридов обнаруживается уже на момент дебюта ПА [18]. Таким образом, повышенный кардиоваскулярный риск при ПА по большей части связан с системным характером иммунного воспаления, а липидные нарушения можно рассматривать как маркер повреждения эндотелия и субклинического атеросклероза. Результаты проведенных исследований позволяют считать дис- и гиперлипидемию косвенным признаком поражения эндотелия и атеросклероза уже на доклинической стадии ПА, не всегда отражающим активность артрита. Эти изменения развиваются рано и длительное время, задолго до клинических симптомов атеросклероза сосудов, остаются незамеченными, а к моменту выявления липидных нарушений у многих пациентов уже имеются проявления ИБС, В т.ч. инфаркт миокарда. Манифестации нарушений липидного обмена способствуют и другие патогенетические звенья сердечно-сосудистых осложнений: ускорение процессов пероксидации и гликирование рецепторов липопротеидов плазмы, нарушения в свертывающей системе и эндотелиальная дисфункция. В результате значительно возрастает суммарный риск развития ССЗ [19]. С целью изучения особенностей течения ПА у больных с дислипидемиями W.H. Boehncke (2010 г.) обследовал 72 пациента. Автор выяснил, что у 76\% больных с дислипидемией IIA типа (гиперхолестеринемия) наблюдался дистальный вариант суставного синдрома, у 82\% с дислипидемией ІІБ типа - полиартритический и спондилоартритический варианты, у 69\% c IV типом дислипидемии - олигоартритический и полиартритический варианты [20]. Можно предположить, что определенный тип дислипидемии также может быть связан с клинической фрормой суставного синдрома ПА.

\section{Повреждение эндотелия и эндотелиальная дисфункция}

Взаимосвязь эндотелиальной дисфункции с особенностями патогенеза ПА и фракторами сердечнососудистого риска - ведущий фактор патогенеза сердечно-сосудистой патологии при данном заболевании. Факторами кардиоваскулярных осложнений для больных ПА также являются молодой возраст начала заболевания, большая длительность и высокая активность артрита [21]. Патогенез кардиоваскулярных осложнений при ПА берет свое начало в повреждении сосудистой стенки. Иммунный ответ у больных ПА сопровождается гиперпродукцией провоспалительных медиаторов, где наибольший интерес представляют медиаторы воспаления - провоспалительные цитокины, матриксные металлопротеиназы, иммунные комплексы и С-реактивный белок, уровень которого определяют в клинической практике для общей оценки активности воспалительного процесса при ПА [21]. На поверхности активированного эндотелия образуются медиаторы, обладающие прокоагулянтными и проатерогенными свойствами, вызывающие отложение липидов в сосудистой стенке и, в последующем, гиперкоагуляцию и образование атеросклеротической бляшки [22]. Можно предположить, что эфффективная терапия ПА способствует подавлению продукции провоспалительных цитокинов, нормализации гемостаза и уменьшению выраженности эндотелиальной дисфункции.

\section{Эхокардиографические изменения}

\section{при псориатическом артрите}

Изменения сосудистой стенки при ПА, обусловленные повреждением эндотелия, подробно изучаются с помощью ультразвукового исследования. По современным данным, сочетание ПА и артериальной гипертензии является состоянием, ассоциированным с повреждением эндотелия [23]. Вовлечение сердечно-сосудистой системы в системное воспаление в виде прогрессирующего атеросклероза отражается не только в изменении липидного профиля, но и в утолщении комплекса интима-медиа (КИМ) артерий. Наиболее удобными для ультразвукового исследования являются сонные артерии. Данные некоторых работ показывают, что для больных ПА характерно раннее развитие атеросклероза - величина комплекса интима-медиа (КИМ) при данной патологии достоверно повышается [23]. О значимом увеличении КИМ при ПА сообщает также Р. Mease (2020 г.), отмечая, что ультразвуковое исследование позволяет выявить его уже на ранней стадии заболевания [24]. По результатам исследования R.B. Mueller и соавт. (2013 г.), среднее значение КИМ в группе больных ПА было значительно выше (0,76 мм), чем в группе контроля (0,64 мм) [25]. Обращает на себя внимание то, что в приведенных исследованиях не была выявлена зависимость толщины КИМ от уровня ЛПНП, ЛПОНП, СРБ, а также наличия артериальной гипертензии, однако изменения КИМ коррелировали с тяжестью ПА, наличием спондилита и не зависели от проводимой противовоспалительной терапии. Это согласуется с данными исследования K. Fitzgerald и соавт. (2012 г.), в котором в группе пациентов с ПА наблюдались достоверно более высокие значения КИМ (0,7 Мм), чем в контрольной группе (0,6 мм), величина КИМ также коррелировала с длительностью заболевания [26]. Мы можем предположить, что изменение толщины КИМ ассоциировано с ПА и является одним из ключевых фракторов поражения сосудистой стенки при данном заболевании.

\section{Нарушения вегетативной регуляции работы сердца у больных ПА}

Хроническое системное воспаление оказывает негативное влияние и на вегетативную регуляцию сердечной деятельности посредством снижения вариабельности сердечного ритма (ВСР) [27]. Последнее, вероятно, можно расценивать как независимый фактор неблагоприятного прогноза и сердечно-сосудистых событий при ПА [27]. По данным клинического исследования А.П. Реброва и соавт. (2011 г.), ухудшение показателей ВСР установлено у всех больных ПА (в исследовании принимали участие только лица, не имеющие 
признаков сердечно-сосудистых заболеваний и соответствующих жалоб). Изменения вегетативной регуляции преимущественно выявляются в виде снижения ВСР и избыточной активации ее симпатического звена, что, безусловно, свидетельствует об отрицательном влиянии системного воспаления на автономную регуляцию работы сердца и отражает напряжение механизмов адаптации сердечно-сосудистой системы [27].

\section{Наиболее часто встречающиеся сердечно-сосудистые} нарушения при ПА

По данным различных авторов, к наиболее распространенным сердечно-сосудистым заболеваниям при ПА относят: гипертоническую болезнь, ишемическую болезнь сердца с/без нарушения ритма, утолщение стенки/дилатацию камер желудочков, приобретенные пороки клапанов, миокардиодистрофию, неревматический миокардит [28]. Артериальная гипертензия (АГ) - один из независимых фракторов риска развития сосудистых осложнений, при ПА встречается чаще, чем в общей популяции [28]. Закономерным следствием повышенного кардиоваскулярного риска, проявляющегося более частым, чем в популяции, проведением аортокоронарного шунтирования, является высокая заболеваемость инфарктом миокарда, составляющая 4,38 на 1000 пациенто-лет [29]. Анализ распространенности кардиоваскулярных заболеваний среди больных ПА был проведен F. Atzeni и соавт. (2013 г.). Согласно результатам исследования гиперлипидемия, клинические проявления атеросклероза и, наконец, частота инфраркта миокарда были достоверно выше при ПА, чем в контрольной группе. Также был выявлен высокий уровень кардиоваскулярной смертности [29]. По данным Y.Z. Tracy и соавт. (2012 г.), относительный риск развития инфаркта миокарда является довольно высоким даже у молодых пациентов. Количество случаев инфраркта миокарда у больных ПА составило 5,13 на 1000 пациенто-лет против 3,58 в контрольной группе [30]. Авторы отмечают более высокую частоту стеноза левой коронарной артерии у больных ПА [30].

\section{Органические изменения сердечно-сосудистой системы при ПА}

Наиболее значимой находкой при ЭХО-КГ у больных ПА является аортит [31]. По данным И. З. Гайдуковой (2011 г.), дилатация аорты и очаговые уплотнения на ее задней стенке, обнаруживаемые у $36 \%$ больных, предположительно ассоциированы с псориатическим спондилитом. Эхокардиографические изменения были выявлены у 21 больного (5,7\%) - аортальная недостаточность превалировала над митральной в 6 раз, комбинированный митрально-аортальный порок встречался вдвое чаще изолированных [31]. F. Atzeni и соавт. (2010 г.) в своем исследовании структуры сердечно-сосудистых нарушений у 30 больных с ПА, не имеющих кардиальных жалоб, получили следующие результаты: у $27 \%$ пациентов была обнаружена диастолическая дисфункция, у 17\% - систолическая дисфункция миокарда левого желудочка, аортальная и митральная недостаточность были выявлены у $37 \%$ и 16,7\% больных соответственно [32]. Эти данные позволяют нам предположить наличие сердечно-сосудистых расстройств у пациентов, не имеющих соответствующих жалоб, что затрудняет своевременную диагностику заболеваний. Крупное российское исследование, проведенное И.З. Гайдуковой (2011 г.), было направлено на выявление зависимости между повреждением эндотелия и кардиоваскулярными осложнениями у 120 больных ПА без клинических проявлений сердечно-сосудистой патологии. По результатам исследования, артериальная гипертензия была выявлена у 55,29\% больных, дислипидемии - у 63\% пациентов с ПА (28\% в группе сравнения), у $34,38 \%$ пациентов выявлено наличие атеросклеротических бляшек. Среди больных с традиционными факторами риска клиническая манифестация ИБС развилась у пациентов с более высокой длительностью и активностью заболевания. По мнению И.З. Гайдуковой, активность ПА напрямую связана с повышением жесткости сосудов [33]. B аналогичном исследовании, проведенном U. Wollina и соавт. (2010 г.), принимали участие 153 больных ПА. Артериальная гипертензия была выявлена у 53\% пациентов (30,9\% в группе контроля), гиперхолестеринемия - 55 и 16,4\%, триглицеридемия - 14,4 и 7,2\% соответственно. Лишь 44,4\% больных ПА принимали гипотензивные препараты (81,8\% - в группе сравнения). Также авторы оценивали риск смерти от ССЗ по шкале SCORE: низкий риск был выявлен у 62\% больных, умеренный - у $17,5 \%$, высокий - у $16,1 \%$, и у $4,7 \%$ больных - очень высокий. В группе сравнения аналогичная оценка составила 83,3, 11,1, 5,6 и 0\% соответственно [34]. Таким образом, артериальная гипертензия у больных ПА обнаруживается на 22,1\% чаще, при этом прием гипотензивных препаратов, напротив, меньше на $37,4 \%$, что еще раз подчеркивает роль рациональной комбинированной терапии в профилактике сердечно-сосудистых осложнений у данных больных.

\section{Оценка кардиоваскулярного риска у больных с ПА}

Повышение сердечно-сосудистой смертности при ПА на популяционном уровне продемонстрировали результаты 4-летнего европейского исследования, которое включало 446 больных со спондилоартритами, в т.ч. ПА [35]. Авторы оценивали риск сердечно-сосудистых осложнений по SCORE и фиксировали их появление через 1 и 4 года. По результатам исследования, появление ИБС и фрибрилляции предсердий у пациентов с ПА было зарегистрировано чаще, чем в группе контроля, риск смерти от СCЗ по шкале SCORE coставил $1 \%$. На первом году и к концу исследования клиника стенокардии была выявлена у 8,3 и 51,2\% пациентов, артериальная гипертензия - у 12,5 и 49\%, инфаркт миокарда развился у 1,6 и 5,8\%, фрибрилляция предсердий - у 1,6 и 7,0\% пациентов соответственно. За время исследования 3 пациента умерли от инфраркта миокарда [35]. По данным М.J. Peters и соавт. (2010 г.), уровень кардиоваскулярного риска (KВP) был средним и выше среднего у большинства больных ПА (55\%) [36].

\section{Заключение}

У больных ПА имеется дисфункция эндотелия, выраженность которой, вероятно, коррелирует со степенью активности данного заболевания. К «классическим» факторам кардиоваскулярного риска у пациентов с ПА добавляются высокая активность заболевания, ранний возраст дебюта ПА, увеличение С-реактивного белка и рентгенологическая деструкция суставов. Можно предположить наличие связи между КВР и утолще- 
нием КИМ сонных артерий, уровнем общего холестерина и ЛПНП у больных ПА. Изучение данной проблемы представляется перспективным направлением в профилактике сердечно-сосудистой смертности у пациентов данной группы. Эффрективная терапия ПА способствует подавлению продукции провоспалительных цитокинов, нормализации гемостаза и уменьшению выраженности эндотелиальной дисфункции. Исходные риски развития сердечно-сосудистых событий у пациентов с ПА превосходят аналогичные риски у лиц, не страдающих данным заболеванием. Своевременное выявление факторов сердечно-сосудистого риска, поиск надежных и современных методов диагностики (дуплексное сканирование сонных артерий, определение циркулирующих эндотелиальных клеток и т.д.) и критериев оценки эндотелиального поражения и атеросклероза с учетом патогенеза ПА продиктовано необходимостью начала ранних мер, направленных на предупреждение/уменьшение смертности от сердечно-сосудистых осложнений при данном заболевании.

\section{Литература/References}

1. Horreau C, Pouplard C, Brenaut E. Cardiovascular morbidity and mortality in psoriasis and psoriatic arthritis: a systematic literature review. J Eur Acad Dermatol Venereol. 2013;27(3):12-29. doi: 10.1111/jdv.12163

2. Schoels MM, Braun J, Dougados M. Treating axial and peripheral spondylarthritis, including psoriatic arthritis, to target: results of a systematic literature search to support an international treat-to-target recommendation in spondylarthritis. Ann Rheum Dis. 2014;73(1):238-242. doi :10.1136/annrheumdis-2013-203860

3. Johnsson H, Mclnnes IB, Sattar N. Cardiovascular and metabolic risks in psoriasis and psoriatic arthritis: pragmatic clinical management based on available evidence. Ann Rheum Dis. 2012;71:480-483. doi: 10.1136/annrheumdis-2011-200567

4. Joep P, Guy De B, Helmut G. European Guidelines on Cardiovascular Disease Prevention in Clinical Practice (Version 2012). Eur J Prev Cardiol. 2012;19(4):585-667. doi: 10.1177/2047487312450228

5. Маркелова Е.И., Коротаева Т.В., Новикова Д.С. и др. Распространенность метаболического синдрома у больных псориатическим артритом: его связь с воспалением и субклиническим атеросклеро30м. Научно-практическая ревматология. 2016;54:20-24. [Markelova El, Korotaeva TV, Novikova DS, et al. The prevalence of metabolic syndrome in patients with psoriatic arthritis: its relationship with inflammation and subclinical atherosclerosis. Scientific and practical rheumatology. 2016;54:20-24 (In Russ.)]

6. Lihi $E$, Arane $T$, Vinod $C$, et al. Increased burden of inflammation over time is associated with the extent of atherosclerotic plaques in patients with psoriatic arthritis. Ann Rheum Dis. 2014;10:1-6.

doi: 10.1136/annrheumdis-2014-205267

7. Дегтярев О.В., Меснянкина О.А. Патогенетическая роль нарушений липидного профиля при псориазе. Российский журнал кожных и венерических болезней. 2015;18(1): 30-33. [Degtyarev OV, Mesnyankina OA. Pathogenetic role of lipid profile disorders in psoriasis. Russian Journal of Skin and Venereal Diseases. 2015;18(1):30-33 (In Russ.)]

8. Ocampo DV, Gladman D. Psoriatic arthritis. F1000Res. 2019;20;8:F1000 Faculty Rev-1665. doi: 10.12688/f1000research.19144.1

9. Favarato $\mathrm{MH}$, Mease $\mathrm{P}$, Gongalves $\mathrm{CR}$, et al. Hypertension and diabetes significantly enhance the risk of cardiovascular disease in patients with psoriatic arthritis. Clin Exp Rheumatol. 2014;32(2):182-187.

10. Гайдукова И.З., Ребров А.П., Лебединская О.А. и др. Кардиоваскулярная заболеваемость и смертность при анкилозирующем спондилите и псориатическом артрите - результаты одноцентрового четырехлетнего наблюдения. Практическая медицина. 2015;2:3(88):123-129. [Gaidukova IZ, Rebrov AP, Lebedinskaya OA, et al. Cardiovascular morbidity and mortality in ankylosing spondylitis and psoriatic arthritis - the results of a single-center four-year follow-up. Practical medicine. 2015;2:3(88):123-129 (In Russ.)]

11. Caso F, Costa L, Chimenti MS, et al. Pathogenesis of Psoriatic Arthritis. Crit Rev Immunol. 2019;39(5):361-377.

doi: 10.1615/CritRevlmmunol.2020033243
12. Holzer $M$, Wolf $P$, Inzinger $M$. Anti-psoriatic therapy recovers high-density lipoprotein composition and function. J Invest Dermatol. 2014;134(3):635-642. doi: 10.1038/jid.2013.359

13. Ахлупкина М.В., Свистунов А.А., Бакулев А.Л. и др. Особенности нарушений в системе цитокинов и липидного обмена у больных псориазом. Саратовский научно-медицинский журнал. 2011;7(2):434437. [Akhlupkina MV, Svistunov AA, Bakulev AL, et al. Features of disorders in the system of cytokines and lipid metabolism in patients with psoriasis. Saratov Journal of Medical Scientific Research. 2011;7(2):434437 (In Russ.)]

14. Arias-Santiago S, Orgaz-Molina J, Castellote-Caballero L, et al. Atheroma plaque, metabolic syndrome and inflammation in patients with psoriasis. Eur J Dermatol. 2012;22(3):337-344. doi: 10.1684/ejd.2012.1714

15. Ritchlin CT, Colbert RA, Gladman DD. Psoriatic Arthritis. N Engl J Med. 2017:9;376(10):957-970. doi: 10.1056/NEJMra1505557

16. Kirby B, Fitzgerald 0 . A new biomarker for psoriatic arthritis? Br J Dermatol. 2020;183(5):805-806. doi: 10.1111/bjd.19141

17. Acebes C., Harvie J. Psoriatic Arthritis. In: El Miedany Y., editors. Musculoskeletal Ultrasonography in Rheumatic Diseases. Springer, Cham. 2015. doi: 10.1007/978-3-319-15723-8_5

18. Badaiki W, Quan Li, Burry TN, Landells F, et al. Predicting Risk of Developing Psoriatic Arthritis (PSA) in Siblings of Patients with Psoriatic Arthritis. Arthritis Rheumatol. 2019;71 (suppl 10).

19. Boehncke S, Salgo R, Garbaraviciene J, et al. Effective continuous systemic therapy of severe plaque-type psoriasis is accompanied by amelioration of biomarkers of cardiovascular risk: results of a prospective Iongitudinal observational study. J Eur Acad Dermatol Venereol. 2011;25(10):1187-1193. doi: 10.1111/j.1468-3083.2010.03947.x

20. Boehncke WH. Managing comorbid disease in patients with psoriasis. BMJ. 2010;340:b5666. doi: 10.1136/bmj.b5666

21. Гайдукова И.3., Ребров А.П. Взаимосвязь жесткости сосудистой стенки и ремоделирования миокарда при псориатическом артрите. Системные ревматические болезни и спондилиты: Материалы ежегодной научно-практической конфреренции. М., 2010;12. [Gajdukova IZ, Rebrov AP. Vzaimosvjaz' zhestkosti sosudistoj stenki i remodelirovanija miokarda pri psoriaticheskom artrite. Sistemnye revmaticheskie bolezni i spondility: Materialy ezhegodnoj nauchno-prakticheskoj konferencii. Moscow, 2010;12 (In Russ.)]

22. Dowlatshahi EA, van der Voort EA, Arends LR, Nijsten T. Markers of systemic inflammation in psoriasis: a systematic review and metaanalysis. Br J Dermatol. 2013;169(2):266-82. doi: 10.1111/bjd.12355

23. Kimball $A B$, Szapary $P$, Mrowietz $U$, et al. Underdiagnosis and undertreatment of cardiovascular risk factors in patients with moderate to severe psoriasis. J Am Acad Dermatol. 2012;67(1):76-85.

doi: 10.1016/j.jaad.2011.06.03

24. Mease P. Preface to the psoriatic arthritis supplement. Rheumatology (0xford). 2020;1;59(Suppl 1):i1-i3. doi: 10.1093/rheumatology/keaa041 
25. Mueller R, Kempis von J. Psoriatic Arthritis. In clinical trials in rheumatology. Springer-Verlag London: 2013. doi: 10.1007/978-1-4471-2870-0_3

26. Fitzgerald K, Hyman M, Swift K. Psoriatic arthritis. Glob Adv Health Med. 2012;1(4):54-61. doi:10.7453/gahmj.2012.1.4.009

27. Ребров А.П., Поддубный Д.А., Гайдукова И.3. Нарушения автономной регуляции сердечной деятельности у больных псориатическим артритом. Клиницист. 2011:2:57-61. [Rebrov AP, Poddubny DA, Gaidukova IZ. Violations of the autonomic regulation of cardiac activity in patients with psoriatic arthritis. Clinician. 2011:2:57-61 (In Russ.)]

28. Boehncke WH, Boehncke S, Tobin AM. The "psoriatic march": a concept of how severe psoriasis may drive cardiovascular comorbidity. Exp Dermatol. 2011;20(4):303-307. doi: 10.1111/j.1600-0625.2011.01261.X.

29. Atzeni F, Turiel M, Boccassini L, et al. Cardiovascular involvement in psoriatic arthritis. Reumatismo. 2011;9;63(3):148-154.

doi: 10.4081/reumatismo.2011.148.

30. Zhu TY, Li EK, Tam LS. Cardiovascular risk in patients with psoriatic arthritis. Int J Rheumatol. 2012;2012:714321.

doi: 10.1155/2012/714321

31. Gaydukova IZ, Poddubnyi DA, Rebrov AP. Heart rate variability in patients with psoriatic arthritis: associations with systemic inflammation and traditional cardiovascular risk factors. Cardiovascular Therapy and Prevention. 2011;10(2):88-92. doi: 10.15829/1728-8800-2011-2-88-92
32. Atzeni F, Straub RH, Cutolo M, Sarzi-Puttini P. Psoriatic arthritis: clinical improvement and correlation with hormone axes in etanercept-treated patients. Ann N Y Acad Sci. 2010;1193:176-178. doi: 10.1111/j.1749-6632.2009.05363.x

33. Гайдукова И.3. Изменение жесткости сосудистой стенки у больных псориатическим артритом при изменении активности заболевания. Материалы ॥ Всероссийского конгресса ревматологов. [Gaidukova IZ. Changes in the rigidity of the vascular wall in patients with psoriatic arthritis with changes in the activity of the disease. Materials of the II All-Russian Congress of Rheumatology. 2011:16 (In Russ.)]

34. Wollina $U$, Unger $L$, Heinig $B$, Kittner $T$. Psoriatic arthritis. Dermatol Ther. 2010;23(2):123-136. doi: 10.1111/j.1529-8019.2010.01306.x

35. Barbarash OL, Kashtalap VV, Shibanova IA. Cardiovascular Comorbidity: Patient with coronary artery disease and peripheral artery atherosclerosis. How to identify and manage the risks of ischemic events? Rational Pharmacotherapy in Cardiology. 2020;16(4):607-613. doi: 10.20996/1819-6446-2020-08-08

36. Peters MJ, Symmons DP, McCarey D, et al. EULAR evidencebased recommendations for cardiovascular risk management in patients with rheumatoid arthritis and other forms of inflammatory arthritis. Ann Rheum Dis. 2010;69(2):325-331. doi: 10.1136/ard.2009.113696

Участие авторов: концепция и дизайн исследования, редактирование, направление на публикацию - 3.Р. Хисматуллина; сбор и обработка материала, написание текста - К.М. Корешкова.

Authors' participation: research concept and design, editing, direction for publication — Zarema R. Khismatullina; collection and processing of material, text writing - Ksenia M. Koreshkova.

\section{Информация об авторах}

*Ксения Михайловна Корешкова - ассистент; адрес: Россия, Республика Башкортостан, 450000, г. Уфра, ул. 50 лет СССР, д. 13, кв. 65; ORCID iD: https://orcid.org/0000-0001-6039-8311; eLibrary SPIN: 4427-1594; e-mail: saitik16@yandex.ru

Зарема Римовна Хисматуллина - д.м.H., профреccop; ORCID iD: https://orcid.org/0000-0001-8674-2803; eLibrary SPIN: 6602-4060; e-mail: hzr07@mail.ru

\section{Information about the authors}

*Ksenia M. Koreshkova - assistant lecturer; address: 13 street 50 years of the USSR, apt. 65, 450000, Ufa, Republic of Bashkortostan, Russia; ORCID iD: https://orcid.org/0000-0001-6039-8311; eLibrary SPIN: 4427-1594; e-mail: saitik16@yandex.ru

Zarema R. Khismatullina - MD, Dr. Sci. (Med.), Professor; ORCID iD: https://orcid.org/0000-0001-8674-2803; eLibrary SPIN: 6602-4060; e-mail: hzr07@mail.ru

Статья поступила в редакцию: 12.01.2021

Принята к публикации: 10.02.2021

Дата публикации: 15.04.2021
Submitted: 12.01 .2021

Accepted: 10.02 .2021

Published: 15.04 .2021 